\title{
Drawing Graphs on a Smartphone
}

\author{
Giordano Da Lozzo, Giuseppe Di Battista, and Francesco Ingrassia \\ Dipartimento di Informatica e Automazione - Università Roma Tre, Italy \\ giordano.dalozzo@gmail.com, gdb@dia.uniroma3.it, fra.ingrassia@gmail.com
}

\begin{abstract}
We present a system for the visualization of relational information on the smartphones. It is implemented on the iPhone and on the Google Android platforms and is based on a new visualization paradigm that poses interesting algorithmic challenges. We also show customizations of the system to explore and visualize popular social networks.
\end{abstract}

\section{Introduction}

Millions of people in the world have in their pocket a smartphone. Such a widely used device is exploited to quickly access, from almost everywhere, different types of data on different subjects. A large amount of such data is relational information. As an example, smartphones are used to access social networks like Facebook or Twitter, ontologies like the Wikipedia network of concepts, or technical information related to the job of the owner like the connections of a computer network or the delivery routes of a goods distribution framework.

Graph Drawing can play an important role in supporting information visualization on the smartphones, provided that the methodologies and tools that are typical of this research area are recast to meet the needs of such a challenging device. Indeed, different information contexts have already changed their visualization methods in this direction. E.g., on-line newspapers have special visualization formats that are designed for the smartphone.

Dealing with smartphones the challenges that visualization applications have to face are, of course, the small screen and the limited memory size. On the other hand, such strong limitations come together with new technological opportunities that can be exploited to support the interaction. They are the multi-touch screen that is able to capture commonly used gestures like pinch, flick, and slide, sensors like the accelerometer and the compass, and sounds and vibrations. All this comes with an always-on connection to the Internet.

A previous attempt to draw graphs on smartphones has been done in [6]. However, such a tool uses Graph Drawing techniques that are not specifically designed for smartphones.

\footnotetext{
* Supported in part by MIUR (Italy), Projects AlgoDEEP no. 2008TFBWL4 and FIRB "Advanced tracking system in intermodal freight transportation", no. RBIP06BZW8.
} 
Since any graph is too large for the little screen of the smartphones, a pivotal reference point for designing interfaces and algorithms for drawing graphs on such a device is the literature on drawing very large graphs. One, for example, could use the fish-eye approach [7] where the details of the drawing decrease according to the distance that separates them from a point chosen by the user. However, using Shneiderman's information visualization mantra 8 (overview first, zoom and filter, then details-on-demand) in this context seems to be unfeasible.

In this paper we present a system for the visualization and interaction with relational information on the smartphones. Section[2 shows the visualization and interaction paradigm we devised, that is based on visualizing a small subgraph defined by a focus vertex and its neighborhood and exploits smartphone-specific interaction primitives. The approach can be considered similar to the navigation approach of [2]. Section 3 shows how even such a simple visualization paradigm can originate interesting algorithmic problems. Section 4 gives details on the adopted algorithms. Section 5 discusses experimental results on such algorithms. Section 6 gives technical details on the implementation and presents case studies.

\section{A Visualization and Interaction Paradigm}

Our visualization paradigm is based on a navigation approach. The user selects a focus vertex $v$ and the drawing contains vertices and edges as follows. Let $N(v)$ be the set of neighbors of $v$, that is assumed circularly ordered. About vertices, the drawing contains $v$ and a subset $\omega_{i}^{L}$, called lobe, of $N(v)$, where $L$ (lobe size), is the size of the lobe and the elements of $\omega_{i}^{L}$ have positions $i, \ldots,(i+L-1)$ $\bmod |N(v)|$ in $N(v)$. About edges, the drawing contains the radial edges from $v$ to the vertices of $\omega_{i}^{L}$, the inner edges between vertices of $\omega_{i}^{L}$, the outer edges that have one end-vertex in $\omega_{i}^{L}$ and one end-vertex in $N(v) \backslash \omega_{i}^{L}$, and the external edges that have one end-vertex in $\omega_{i}^{L}$ and one end-vertex that is not in $N(v)$. The focus vertex is in the center of the bottom side of the drawing, while the lobe vertices lie on an half-ellipse centered at the focus vertex. This, together with other graphical features, suggests to the user that $\omega_{i}^{L}$ is only a subset of $N(v)$ and that the vertices of $N(v) \backslash \omega_{i}^{L}$ (outer vertices) are under the bottom side of the screen. External edges exit the drawing from the left, top, right borders of the screen, giving the impression that the rest of the graph is outside the screen. Their external end-vertices are not represented. Outer edges are directed downward, leading to vertices of $N(v)$ that are not represented and that are under the bottom side of the screen. Fig. 1 shows an example of visualization.

Different types of edges have different thicknesses. Radial edges, that do not give additional information, are thin, while inner edges, that represent relationships between vertices of the lobe, are emphasized. External edges could be too many to be represented explicitly, hence they are drawn with straight-lines only up to a certain number. Over that number they are shown with a shadow exiting the drawing. Essentially, the important information for the user is if they are 0, a few, or a lot. Similar graphic features are used for outer edges. 

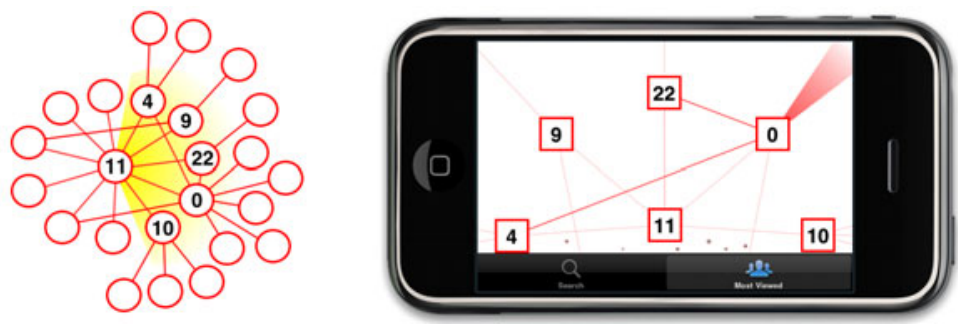

Fig. 1. Visualization of the subgraph induced by the lobe plus the focus vertex (vertex 11)

The user interacts with the graph according to the following primitives. There are vertex oriented primitives like focus change, navigation backtrack, and execution of actions associated with vertices (e.g., open a browser or send an sms) and lobe oriented primitives like lobe shift, lobe resize, and lobe layout temporary optimization.

The focus change primitive substitutes the currently visualized subgraph with the subgraph induced by a new focus vertex and its neighborhood. The gesture used to change the focus consists of dragging the vertex of the lobe that will be the new focus towards the focus position. The backtrack primitive shows the previously displayed lobe. The user can navigate the path of the explored focus vertices by performing a double fingers flick towards the bottom or top side of the screen. A side feature of our framework is the possibility of extending the interaction experience by executing an action related to a selected vertex. For example, if the input graph is a social network, possible actions are: showing more information about the individual, sending an e-mail or an sms, deleting him/her from the graph, etc. The gesture associated with this action is a double tapping or a pressure on the vertex.

A primitive that changes the lobe is lobe shift. Let $\omega_{i}^{L}$ be the visualized lobe, the effect of lobe shifting is to substitute $\omega_{i}^{L}$ with $\omega_{k}^{L}$ where $k=i \pm 1 \bmod |N(v)|$. Sliding a finger on the screen is the simpler way to do this. However, if $|N(v)|$ is very large this interaction can be unsuitable to reach vertices that are far from the current lobe. Hence, the user needs a single gesture to skip a large portion of the subgraph. This is obtained with a flick of finger on the screen. The number of the vertices that are skipped is proportional to the speed of the gesture. The same primitive can be invoked using the accelerometer by changing the slope of the device or using the compass by changing the orientation of the device.

Zooming is a quite common functionality offered by smartphone applications. In our paradigm this function corresponds to a lobe resize. Let $\omega_{i}^{L}$ be the visualized lobe, the effect of this function is to show the lobe $\omega_{k}^{M}$ where $k=i$ if $(M=L) \vee(M=L+1)$ or $k=i-1 \bmod |N(v)|$ if $M=L+2$. The gesture used to resize the current lobe is a multi-touch gesture called pinch, this consists of moving together two fingers on the screen, increasing or decreasing their distance. 
The current order of the vertices of a lobe could lead to a drawing that is unpleasant. Hence, we provide a local optimization feature that temporary reorders the vertices of the lobe in order to increase the readability of the drawing. This local optimization is invoked by performing a double tap on the screen.

In order to help the user in maintaining the mental map during the navigation, a mechanism for morphing between successive drawings, supported by a smooth movement of edges and vertices, is necessary. Hence, for all primitives, vertices move slowly towards their final positions. In lobe oriented primitives, vertices entering (exiting) the current lobe come in (out) from the bottom side of the screen. Also, inner edges can become external and vice-versa, and their representation features change coherently. The flick function is accompanied by an inertial rotation of the neighborhood of the focus vertex. The morphing features of the focus change primitive are more complex than those described above. Since they depend on the order of the lobes, they are discussed in Section 4. With the purpose of helping the user interaction experience, sounds and vibration events are associated with the primitives. They are selected in such a way to be consistent with the effects of the corresponding primitives.

\section{Choosing the Lobe Order}

An important aspect of the visualization paradigm is the left-to-right order of the vertices of the lobe. The specific choice of this order may depend on the specific application. However, the need of preserving the user's mental map implies that the orderings of contiguous lobes of a focus vertex $v$ are consistent. Since this constraint holds for all the lobes of $v$, this implies the need of choosing a unique order for all the vertices of $N(v)$.

Trivial choices are possible, like using the alphabetical order, that can be suitable for some applications. On the other hand, it is also possible to make different choices, according to aesthetics that are related to the selected visualization paradigm. We deepen two different choices corresponding to two aspects of the paradigm. The first is the one of displaying the relational information as clean as possible, while the second is the one of showing as much relational information as possible. The two choices conflict each other.

The first choice is the one of selecting an order that tries to minimize the visible crossings. Given a focus vertex and a lobe, a visible crossing is a crossing between two inner edges. We concentrate only on visible crossings because the only edges that are completely visible for a certain lobe are the inner edges and the radial edges. Also, crossings with radial edges do not compromise readability. More precisely, we try to minimize the average number of visible crossings for all the lobes of a focus vertex.

The problem of minimizing the visible crossings has similarities with the circular crossings minimization problem. In that problem, the vertices lie on a circumference, the edges are straight lines, and a circular order is searched that minimizes the total number of crossings. See, e.g. 4913]. However, as shown in Fig. 2, the two problems are different. 


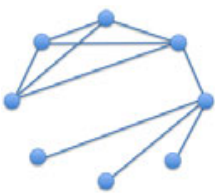

(a)

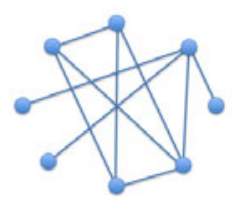

(b)

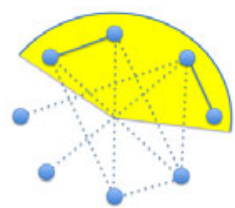

(c)

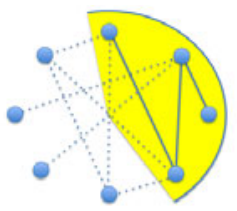

(d)

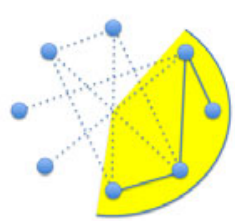

(e)

Fig. 2. Circular crossings and visible crossings. (a) Order with minimum total number of crossings. (b) Order with minimum number of visible crossings if the lobe size is equal to 4 . (c)-(e) Lobes with 0 visible crossings.

Observe that minimizing the visible crossings with a lobe with size that is equal to $|N(v)|$ is equivalent to solving a circular crossings minimization on the subgraph induced by $N(v)$. But, unfortunately, such a problem has been proved NP-complete 5]. Hence, solving in practice the minimization of the visible crossings requires the usage of heuristics.

One may ask whether heuristics for the circular crossings minimization are effective also for our problem. Our experiments show that this is not the case (see Section 5). Hence, we used in our system a special purpose algorithm that is described in Section 4 .

A second alternative for choosing an order is to select one that tries to maximize the visible edges, that is to minimize the number of edges that are not inner edges in any lobe. This corresponds to minimizing the information that is lost for a certain focus vertex. Let $L$ be the lobe size and let $v$ be the focus vertex. Observe that it might not exist an order for $N(v)$ in which every edge with end-vertices in $N(v)$ is an inner edge for at least one lobe of size $L$. As an example, consider the case when the subgraph induced by $N(v)$ contains the clique $K_{M}$ with $M \geq 2 L$. Of course, this information is not lost. In fact, such edges will be visible selecting one of the end-vertices of the missing edges as focus vertex. Observe the similarities with the Graph Bandwidth Problem.

\section{Algorithmic Framework}

The algorithmic framework that we have developed in our system allows to tackle both the visible crossings minimization and the visible edges maximization problems.

The typical parameters of the algorithms for the circular crossings minimization problem presented in 4113 are: 1 . the start vertex $s$ of the graph to be drawn; 2. the policy which selects the next vertex to process; and 3 . the position that is chosen for the selected vertex. We make use of the same general setting. The algorithmic framework we propose is Algorithm 1 .

Notice that Algorithm 1 refers to a vertex processing policy that is not specified. The main processing policies used in the literature for determining the insertion sequence are: Random, vertices are processed in random order; Maximum degree, at each step, a vertex with the largest number of neighbors is 


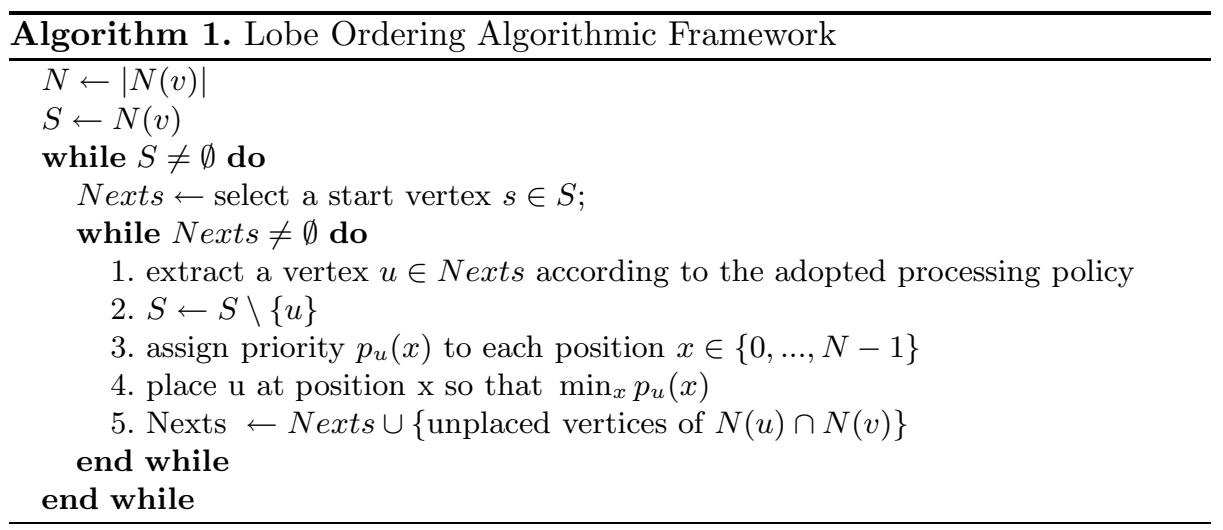

placed; Minimum degree, at each step, a vertex with the least number of neighbors is placed; and Connectivity, at each step, a vertex with the largest number of already placed neighbors and (in case of ties) the least number of unplaced neighbors is selected. In our experiment we test the effectiveness of each of them.

Note that the inner cycle of Algorithm 11 involves vertices belonging to the same connected component. Indeed, inside this cycle, a wave-like scanning of the connected component is performed. The outer cycle is used to iterate the inner one on all the connected components, until all vertices are placed.

In order to evaluate the impact (in terms of the qualities of interest) of the placement of the selected vertex in each available position of the current layout, we introduce a real valued priority function. The function $p_{u}(x):\{0 \ldots N-$ $1\} \rightarrow \mathbb{R}$ estimates the quality of the position $x$ for vertex $u$ considering the cost associated with the edges that link $u$ to its already placed neighborhood (i.e. $\left.N_{p}(u)\right)$. We define length $(s, t)=\min (|s-t|, N-|s-t|)$. Let $p_{u}(x)=$ $\sum_{w \in N_{p}(u)} c_{k}($ length $(x, \pi(w)), L)$ if position $x$ is available and let $p_{u}(x)=\infty$ otherwise.

With reference to the qualities desired for the representation, we can define different cost functions $c_{k}(d, L):\{1, \ldots,\lfloor N / 2\rfloor\} \times\{1, \ldots, N\} \rightarrow \mathbb{R}$ that estimate the burden produced by an edge of length $d$ in a layout with lobe size $L$. Fig. 3 shows two possible cost functions aimed at minimizing the number of crossings between inner edges. The former function $c_{L}(d, L)$ (MinLobe) estimates the cost of an edge of length $d$ in a lobe of size $L$ as the maximum number of visible crossings this edge can produce if there exist (in the lobe) all the edges that are able to cross it. The latter function $c_{L S}(d, L)$ (MinLobeSum) computes a weighted average of the previous one with respect to the number of lobes the edge will appear in.

Actually, the current implementation of the algorithm associates with each location a list of priority values that are sequentially evaluated. This feature makes it possible to choose the final position of a vertex using multiple selection criteria (or, equivalently, a multi-objective function), whose relative importance is defined by the order they are taken into account. 

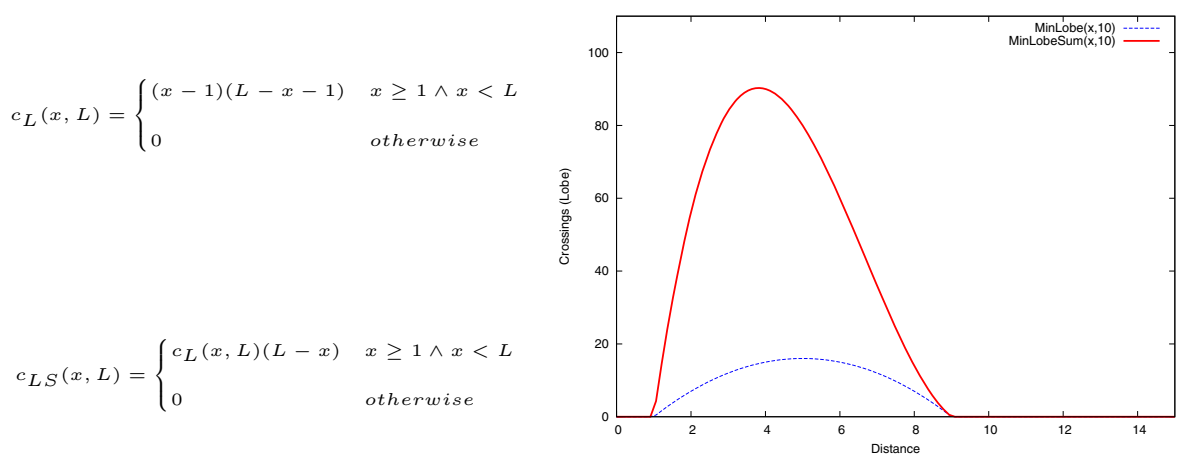

Fig. 3. Cost functions for minimizing visible crossings

If we want to maximize the visible edges, a simple cost function whose purpose is to maximize the number of edges that can become inner edges in some lobe is function $c_{I}(d, L)$ (MaxVisibleEdge), defined as follows: $c_{I}(d, L)=-1$ if $d<L$ and $c_{I}(d, L)=0$ if $d \geq L$.

If we find that two positions have the same priority, we break the tie using a cost function called MinEdgeLength, that is $c_{D}(x, L)=x \forall x, L$. It is used to avoid enlarging too much the set of considered positions.

As we said in Section 2] special attention is needed to perform an effective morphing procedure for the focus change primitive from the current focus $v$ to the new focus $u$. This has important effects also in the algorithmic framework. Let $\omega_{i}^{L}$ be the visible lobe of $v$. It is essential, to preserve the user mental map, to order the vertices in $N(u)$ such that the relative order of the vertices in $N(u) \cap \omega_{i}^{L}$ remains the same. Also, the external vertices of $u$ that enter the drawing must be placed after the inner vertices at the left of $v$ in the lobe. Hence, the algorithm should be able to impose constraints of the above type on the lobe ordering. Observe that the above algorithmic approach, since the positions are explicitly represented, easily integrates such type of constraints.

\section{Experimental Analysis of the Algorithms}

In order to asses the effectiveness of our algorithmic techniques we performed several experimental tests using a suite of randomly generated connected graphs, where each graph represents the subgraph induced by $N(v)$ for some choice of focus vertex $v$. For each test we generated an average of 5,000 graphs according to two modalities: 1 . fixed number of vertices (100) and variable density (from $0.1 \%$ to $100 \%$ ); 2 . fixed density and variable number of vertices (from 10 to 100). Both types of graphs are evaluated with a fixed lobe size of 10 . This value represents a good compromise between the number of the visible vertices and an effective usage of the available space. 
We evaluated the algorithms according to the following requirements: 1 . visible crossings, that is the number of crossings between inner edges; 2 . visible edges ratio, that is the ratio between the number of visible edges and the number of edges of the graph. A selection of the results is presented below.

In the experiment shown in Fig. 4(a) we compare a simple random order with the BB algorithm [1], chosen as one of the best algorithms for computing a circular layout with few crossings. Each point shows the average number (computed over all lobes) of the visible crossings for a graph whose density is reported on the $x$-axis. A random order produces fewer visible crossings than the BB algorithm. Such results suggest that the circular approach is not a good choice for the visible crossings minimization problem. The tested circular algorithm tries to minimize the total length of the edges but short edges enter more easily in a lobe, increasing the possibility of generating crossings.

In the experiment shown in Fig. 4(c) we compare several variants of our algorithm (see Fig. 6). We changed the processing policy that selects the next vertex to insert (Random or Connectivity) and the cost functions for each level of priority (MinLobe or MinLobeSum). Observe that in all cases we use, for a second priority level, cost function MinEdgeLength. Mixing these features we obtain four different algorithms to evaluate (RLobe, RSum, CLobe, CSum). The graphic shows the comparison between these algorithms with a variable number of vertices and fixed density $15 \%$. It is evident that the choice of a good processing policy has a strong influence on the number of visible crossings. Algorithms that use the connectivity policy perform better than those using the random policy. For a given processing policy, the MinLobeSum cost function is slightly better than the MinLobe.

In the experiment shown in Fig. 4(e) we compare the average number of visible crossings of the CSum algorithm with the minimum number of visible crossings on a lobe of a Random placement. The graphic shows that CSum has a similar trend to Random with slightly better results. Roughly, given an unordered layout and selected the lobe with lower number of visible crossings, the algorithm generates a drawing with the same average number of visible crossings for each lobe.

In the experiment shown in Fig. 4(b) we compare the visible edges ratio between Random order and BB Algorithm. Although BB is not designed for solving this problem, drawing shorter edges decreases the number of edges hidden by the paradigm and therefore increases the visible edges ratio. In the graphic we observe that BB Algorithm has a better behavior than the one of a Random order.

For this problem we follow two different approaches. The first is to reverse the cost function of CSum in order to increase the number of crossings and consequently the number of visible edges. The second approach is a typically greedy approach where a vertex is positioned in the local highest visible edges position. This cost function is associated with a Random and Connectivity processing policy. A Random selection decreases the effectiveness of the results of 
the MaxVisibleEdge cost function. With an equal processing policy the MaxVisibleEdge function is slightly better than MaxSum. (See Fig. 4(d),

In the experiment shown in Fig. 4(f) we compare CVis algorithm with BB algorithm. CVis has better results than BB, especially with a small number of vertices (CVis keeps visible $100 \%$ of edges for graphs with more vertices than $\mathrm{BB})$. Increasing the number of vertices both algorithms have the same trend and CVis has generally an improvement of $2-3 \%$.

\section{Implementation and Case Studies}

The project has been fully driven by the experiments performed on the devices. (See in Fig. 5(a) and 5(b) the usage of the important primitive focus change over the iPhone device.) This led to two SW libraries, one for the iPhone OS 3.1.3 (Objective-C language) and the other for the Google Android 2.1 (Java language) platforms. Both the libraries were designed to have fully customizable graphical and behavioral components and to allow simple usage for the SW developer.

Although the two platforms are very different, we developed the SW so that both prototypes have the following common features: 1. use vector graphics, 2. build a new abstract layer over the platform to manage animations and gestures, and 3. optimize the number of operations and edge drawings needed for each display refresh. Because of the limitations of the platform, the Android release required also to minimize the number of refreshes.

We implemented several case studies. Two of them, that refer to the context of social networks, are especially interesting.

The first case study uses the Facebook API to determine the graph of the friendships of a Facebook user (see Fig. 5(c) and 5(d)]. These APIs allow to read (and write) objects and social connections of the Facebook Graph. The objects, that are the vertices of the graph, have a unique identifier (ID) and their associated data can be retrieved with a simple fetch of the URL https:// graph.facebook.com/ID. All objects are linked together through relationships of different types for different objects. We retrieve the connections using URLs of the form: https://graph.facebook.com/ID/CONNECTION_TYPE. Obviously, in order for the queries to succeed they must not violate the privacy restrictions set by the users. The user can provide his/her personal credentials (username and password) to our FacebookView Application through an input window. The system automatically generates the necessary requests to determine the subgraph of the Facebook Graph induced by the set of vertices consisting of the facebook user and his/her friends. Actually, in the current implementation the set of vertices (and relationships between them) is extended at run-time with queries that refer to resources (events, photos, links, videos, etc.) liked or tagged by other users.

The second case study shows the public relations exposed and available on the Web from the Google Social Graph API (see Fig. 5(f). This information 


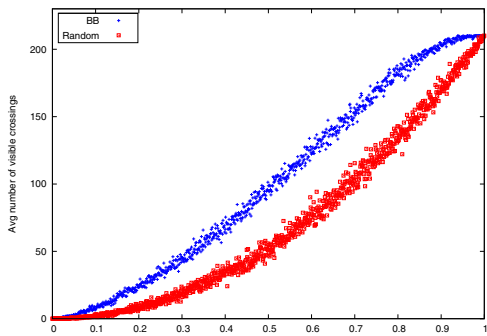

(a) $|N(v)|=100, \mathrm{~L}=10$.

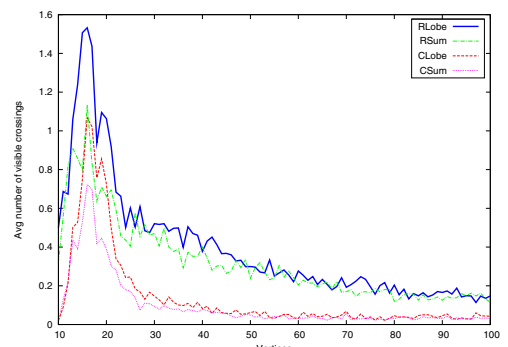

(c) $|N(v)|=\{10, \ldots, 100\}, \mathrm{L}=10,(\mathrm{~d})|N(v)|=100, \mathrm{~L}=10$, density $=$ density $=15 \%$.

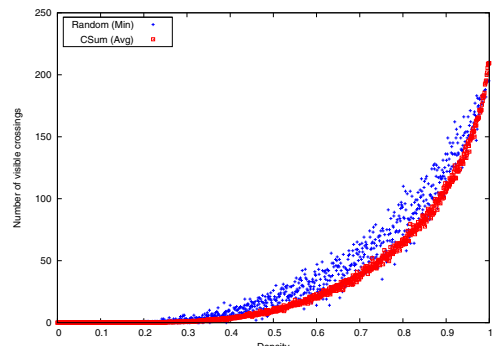

(e) $|N(v)|=100, \mathrm{~L}=10$, density $=0-($ $100 \%$.

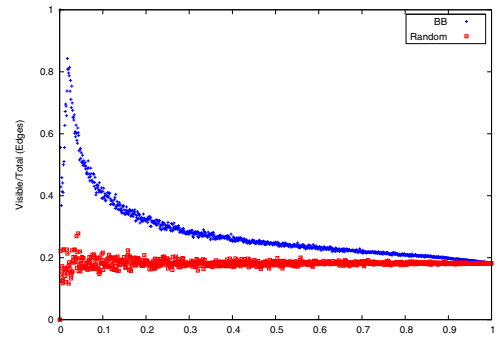

(b) $|N(v)|=100, \mathrm{~L}=10$.

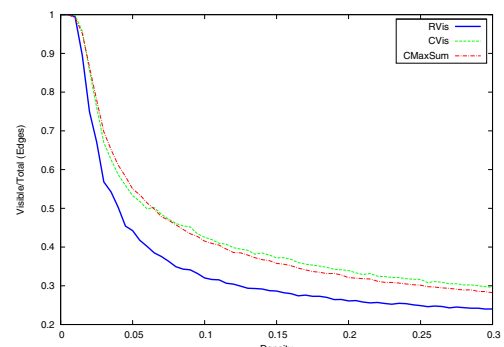
$0-30 \%$.

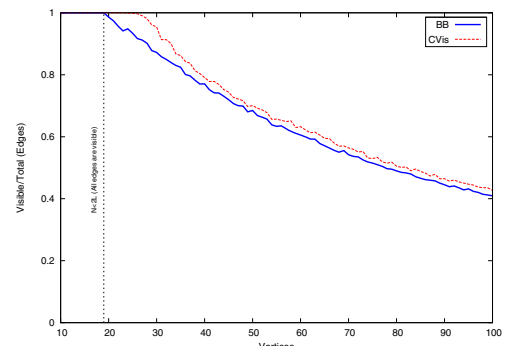

(f) $|N(v)|=\{10, \ldots, 100\}, \quad \mathrm{L}=10$, density $=15 \%$.

\begin{tabular}{|c|c|c|}
\hline Algorithm & Processing policy & Cost functions \\
\hline RSum & Random & $\begin{array}{l}\text { 1: MinLobeSum } \\
\text { 2: MinEdgeLength }\end{array}$ \\
\hline RLobe & Random & $\begin{array}{l}\text { 1: MinLobe } \\
\text { 2: MinEdgeLength }\end{array}$ \\
\hline CSum & Connectivity & $\begin{array}{l}\text { 1: MinLobeSum } \\
\text { 2: MinEdgeLength }\end{array}$ \\
\hline CLobe & Connectivity & $\begin{array}{l}\text { 1: MinLobe } \\
\text { 2: MinEdgeLength }\end{array}$ \\
\hline$\overline{\mathrm{CV} \text { is }}$ & Connectivity & MaxVisibleEdge \\
\hline CMaxSum & Connectivity & $\begin{array}{l}\left.\text { 1: MaxSumLobe (i.e. }-c_{L S}(x, L)\right) \\
\text { 2: MinEdgeLength }\end{array}$ \\
\hline
\end{tabular}

Fig. 4. Algorithms experimental results 


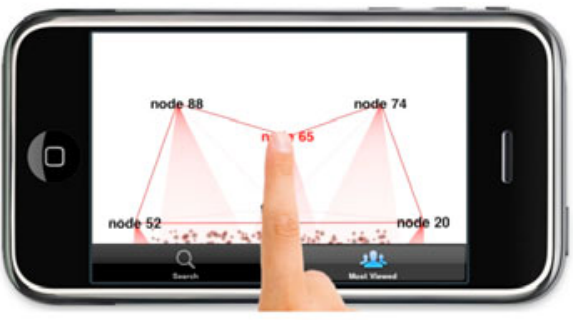

(a) A focus change starts

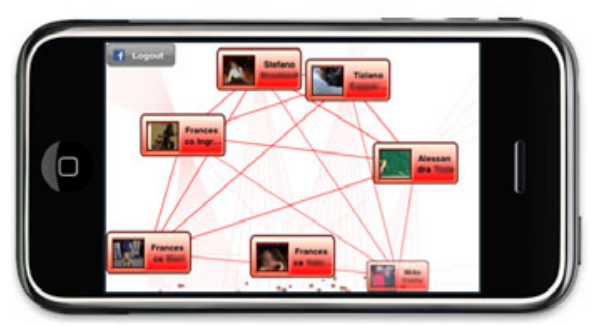

(c) FacebookView over iPhone

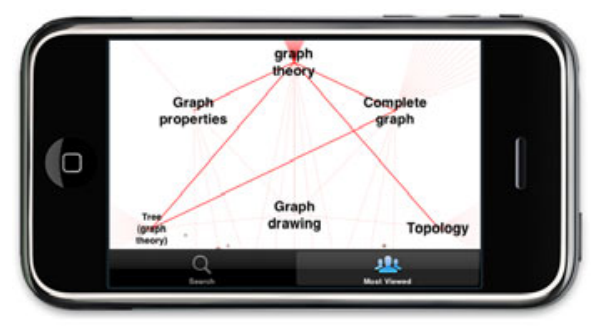

(e) WikipediaView over iPhone

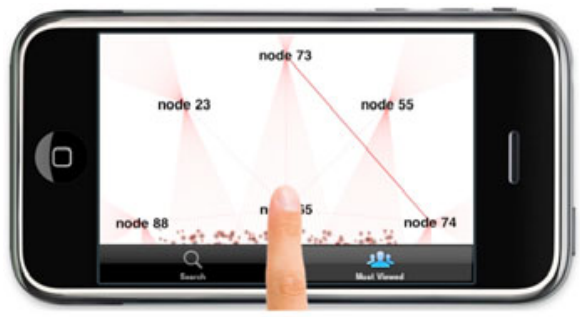

(b) Focus change result

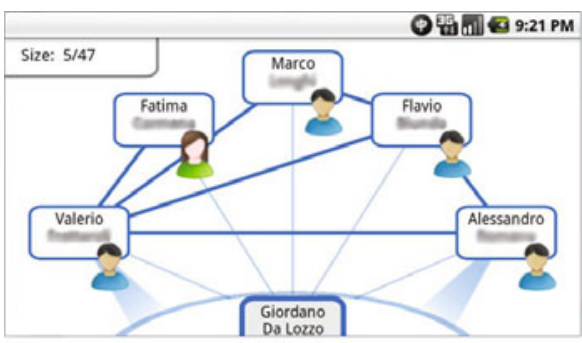

(d) FacebookView over Android

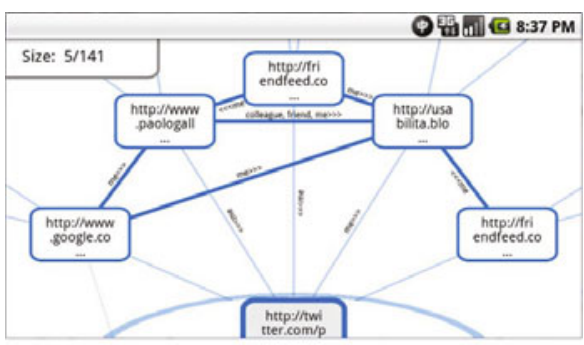

(f) SocialView over Android

Fig. 5. Application samples

is declared within public profiles via XFN (XHTML Friends Network), FOAF (Friend Of A Friend), and other declared public connections. For example, XFN provides a simple way to define human relationships through Web links using the $r e l$ attribute of the $<a$ href $>$ tag (e.g. < a href= "http://pino.example.com/" rel= "colleague met" $>$ Pino $</ \mathrm{a}>$ ). The user can provide to our SocialView Application the URL of a public account through an input window. The system automatically generates a query for acquiring as much relational information as possible.

We also implemented a case study to explore Wikipedia (see Fig. 5(e)]. A user selects a word and the smartphone shows the related concepts. For example if the user selects Graph Drawing the device shows the Graph Theory, Topology, Geometry, etc. When the user finds an interesting concept, he/she can expand the vertex and visualize the Wikipedia page. 


\section{References}

1. Baur, M., Brandes, U.: Crossing reduction in circular layouts. In: Hromkovič, J., Nagl, M., Westfechtel, B. (eds.) WG 2004. LNCS, vol. 3353, pp. 332-343. Springer, Heidelberg (2004)

2. Eades, P., Cohen, R.F., Huang, M.L.: Online animated graph drawing for web navigation. In: di Battista, G. (ed.) GD 1997. LNCS, vol. 1353, pp. 330-335. Springer, Heidelberg (1997)

3. He, H., Sykora, O.: New circular drawing algorithms. In: Proc. ITAT 2004 (2004)

4. Makinen, E.: On circular layouts. Internal Journal of Computer Mathematics, 29-37 (1988)

5. Masuda, S., Kashiwabara, T., Nakajima, K., Fujisawa, T.: An NP-hard crossing minimization problem for computer network layout. Technical report (1986)

6. Pixelglow. Instaviz (2008), http://instaviz.com/

7. Sarkar, M., Brown, M.H.: Graphical fisheye views of graphs. In: Proceedings of the Conference on Human Factors in Computing Systems CHI 1992 (1992)

8. Shneiderman, B.: The eyes have it: A task by data type taxonomy for information visualizations. In: Proc. of the IEEE Symp. on Visual Lang., pp. 336-343 (1996)

9. Six, J.M., Tollis, I.G.: Circular drawings of biconnected graphs. In: Proc. ALENEX (1999) 